\title{
Correction to: Adjunctive Brivaracetam in Focal Epilepsy: Real-World Evidence from the BRIVAracetam add-on First Italian netwoRk Study (BRIVAFIRST)
}

\author{
Simona Lattanzi ${ }^{1}$ - Laura Canafoglia ${ }^{2}$. Maria Paola Canevini ${ }^{3,4} \cdot$ Sara Casciato ${ }^{5}$. Valentina Chiesa ${ }^{3}$. \\ Filippo Dainese $^{6}$. Giovanni De Maria ${ }^{7}$. Giuseppe Didato ${ }^{8}$. Giovanni Falcicchio ${ }^{9} \cdot$ Martina Fanella $^{10}$. \\ Edoardo Ferlazzo ${ }^{11}$. Giacomo Fisco ${ }^{10}$. Massimo Gangitano ${ }^{12}$. Anna Teresa Giallonardo ${ }^{10}$. Filippo Sean Giorgi ${ }^{13,14}$. \\ Angela La Neve ${ }^{9}$. Oriano Mecarelli ${ }^{10}$. Elisa Montalenti ${ }^{15} \cdot$ Federico Piazza $^{16}$. Patrizia Pulitano ${ }^{10}$. \\ Pier Paolo Quarato ${ }^{5}$. Federica Ranzato ${ }^{17}$. Eleonora Rosati ${ }^{18}$. Laura Tassi ${ }^{19} \cdot$ Carlo Di Bonaventura $^{10}$ on behalf of \\ BRIVAFIRST Group Membership
}

Published online: 17 November 2021

(c) The Author(s) 2021

\section{Correction to: CNS Drugs https://doi.org/10.1007/s40263-021-00856-3}

In the original publication, the names of the BRIVAFIRST group members were incorrectly presented and tagged in the Acknowledgements section. This has now been corrected as follows.

The original article can be found online at https://doi.org/10.1007/ s40263-021-00856-3

Simona Lattanzi

alfierelattanzisimona@gmail.com

1 Neurological Clinic, Department of Experimental and Clinical Medicine, Marche Polytechnic University, Via Conca 71, 60020 Ancona, Italy

2 Department of Epileptology, Fondazione IRCCS Istituto Neurologico Carlo Besta, Milan, Italy

3 Epilepsy Center, Child Neuropsychiatry Unit, AAST Santi Paolo Carlo, Milan, Italy

4 Department of Health Sciences, Università degli Studi, Milan, Italy

5 IRCCS Neuromed, Pozzilli, Italy

6 Epilepsy Centre, Neurology Unit, Venice, Italy

7 Clinical Neurophysiology Unit, Epilepsy Center, Spedali Civili, Brescia, Italy

8 Epilepsy Unit, Fondazione IRCCS Istituto Neurologico "Carlo Besta", Milan, Italy

9 Department of Basic Medical Sciences, Neurosciences and Sense Organs, University Hospital of Bari "A. Moro", Bari, Italy
Acknowledgements The authors would like to thank the participants of the BRIVAFIRST group for contributing their data for research.

BRIVAFIRST Group Membership Angela Alicino ${ }^{9}$, Michele Ascoli ${ }^{11}$, Giovanni Assenza ${ }^{20}$, Federica Avorio ${ }^{10}$, Valeria Badioni ${ }^{21}$, Paola Banfi ${ }^{22}$, Emanuele Bartolini ${ }^{23}$, Luca Manfredi Basili ${ }^{10}$, Vincenzo Belcastro ${ }^{24}$, Simone Beretta ${ }^{25}$, Irene Berto ${ }^{10}$, Martina Biggi $^{18}$, Giuseppe Billo ${ }^{17}$, Giovanni Boero ${ }^{26}$, Paolo Bonanni ${ }^{27}$, Jole Bongorno ${ }^{28}$, Francesco Brigo ${ }^{29}$, Emanuele Caggia ${ }^{28}$, Claudia Cagnetti $^{1}$, Carmen Calvello ${ }^{30}$, Emanuele Cerulli Irelli ${ }^{10}$, Edward Cesnik $^{31}$, Gigliola Chianale ${ }^{32}$, Domenico Ciampanelli ${ }^{33}$, Roberta Ciuffini $^{34}$, Dario Cocito ${ }^{35}$, Donato Colella ${ }^{10}$, Margerita Contento ${ }^{18}$, Cinzia Costa ${ }^{30}$, Eduardo Cumbo ${ }^{36}$, Alfredo D'Aniello ${ }^{5}$, Francesco Deleo $^{8}$, Jacopo C DiFrancesco ${ }^{25}$, Giancarlo Di Gennaro ${ }^{5}$, Roberta Di

10 Department of Human Neurosciences, Policlinico Umberto I, Sapienza University of Rome, Rome, Italy

11 Department of Medical and Surgical Sciences, Magna Græcia University of Catanzaro, Catanzaro, Italy

12 Department of Biomedicine, Neuroscience, and advanced Diagnostic (BIND), University of Palermo, Palermo, Italy

13 Department of Translational Research on New Technologies in Medicine and Surgery, University of Pisa, Pisa, Italy

14 Neurology Unit, Pisa University Hospital, Pisa, Italy

15 Epilepsy Center, AOU Città della Salute e della Scienza di Torino, Turin, Italy

16 Department of Neurosciences, Rita Levi Montalcini, University of Turin, Turin, Italy

17 Epilepsy Center, UOC Neurology, AULSS, 8 Vicenza, Vicenza, Italy

18 Department Neurology 2, Careggi University Hospital, Florence, Italy

19 “C. Munari” Epilepsy Surgery Centre, Niguarda Hospital, Milan, Italy 
Giacomo $^{8}$, Alessandra Di Liberto ${ }^{32}$, Elisabetta Domina ${ }^{21}$, Francesco Donato $^{6}$, Fedele Dono ${ }^{37}$, Vania Durante ${ }^{38}$, Maurizio Elia ${ }^{39}$, Anna Estraneo $^{40}$, Giacomo Evangelista ${ }^{37}$, Maria Teresa Faedda ${ }^{10}$, Ylenia Failli $^{18}$, Elisa Fallica ${ }^{31}$, Jinane Fattouch ${ }^{10}$, Alessandra Ferrari ${ }^{41}$, Florinda Ferreri ${ }^{42}$, Davide Fonti ${ }^{43}$, Francesco Fortunato ${ }^{44}$, Nicoletta Foschi ${ }^{1}$, Teresa Francavilla ${ }^{9}$, Rosita Galli ${ }^{45}$, Stefano Gazzina ${ }^{7}$, Loretta Giuliano ${ }^{46}$, Francesco Habetswallner ${ }^{47}$, Francesca Izzi ${ }^{48}$, Benedetta Kassabian ${ }^{42}$, Angelo Labate ${ }^{44}$, Concetta Luisi ${ }^{42}$, Matteo Magliani ${ }^{18}$, Giulia Maira ${ }^{46}$, Luisa Mari ${ }^{48}$, Daniela Marino ${ }^{45}$, Addolorata Mascia ${ }^{5}$, Alessandra Mazzeo ${ }^{33}$, Stefano Meletti ${ }^{49}$, Alessandra Morano $^{10}$, Annacarmen Nilo ${ }^{50}$, Biagio Orlando ${ }^{10}$, Francesco Paladin ${ }^{6}$, Maria Grazia Pascarella ${ }^{21}$, Chiara Pastori ${ }^{8}$, Giada Pauletto ${ }^{51}$, Alessia Peretti $^{17}$, Gabriella Perri ${ }^{52}$, Marianna Pezzella ${ }^{47}$, Marta Piccioli ${ }^{53}$, Pietro Pignatta ${ }^{54}$, Nicola Pilolli ${ }^{26}$, Francesco Pisani ${ }^{55}$, Laura Rosa Pisani $^{56}$, Fabio Placidi ${ }^{48}$, Patrizia Pollicino ${ }^{57}$, Vittoria Porcella ${ }^{58}$, Silvia Pradella ${ }^{23}$, Monica Puligheddu ${ }^{43}$, Stefano Quadri ${ }^{59}$, Rui Quintas ${ }^{8}$, Rosaria Renna ${ }^{60}$, Jessica Rossi ${ }^{49}$, Adriana Rum ${ }^{61}$, Enrico Michele Salamone $^{10}$, Ersilia Savastano ${ }^{10}$, Maria Sessa ${ }^{59}$, David Stokelj ${ }^{62}$, Elena Tartara $^{63}$, Mario Tombini' ${ }^{20}$, Gemma Tumminelli ${ }^{3}$, Maria Ventura ${ }^{28}$, Ilaria Viganò ${ }^{3}$, Emanuela Viglietta ${ }^{54}$, Aglaia Vignoli ${ }^{4}$, Flavio Villani ${ }^{41}$, Elena Zambrelli ${ }^{3}$, Lelia Zummo ${ }^{64}$

Affiliations

1. Neurological Clinic, Department of Experimental and Clinical Medicine, Marche Polytechnic University, Ancona, Italy

2. Department of Epileptology, Fondazione IRCCS Istituto Neurologico Carlo Besta, Milan, Italy

3. Epilepsy Center, Child Neuropsychiatry Unit, AAST Santi Paolo Carlo, Milan, Italy Italy

4. Department of Health Sciences, Università degli Studi, Milan,

5. IRCCS Neuromed, Pozzilli, Italy

6. Epilepsy Centre, Neurology Unit, Venice, Italy

7. Clinical Neurophysiology Unit, Epilepsy Center, Spedali Civili, Brescia, Italy

8. Epilepsy Unit, Fondazione IRCCS Istituto Neurologico "Carlo Besta", Milan, Italy

9. Department of Basic Medical Sciences, Neurosciences and Sense Organs-University Hospital of Bari "A. Moro"

10. Department of Human Neurosciences, Policlinico Umberto I, Sapienza University of Rome, Italy

11. Department of Medical and Surgical Sciences, Magna Græcia University of Catanzaro, Catanzaro, Italy

12. Department of Biomedicine, Neuroscience, and advanced Diagnostic (BIND), University of Palermo, Palermo, Italy

13. Department of Translational Research on New Technologies in Medicine and Surgery, University of Pisa, Pisa, Italy

14. Neurology Unit, Pisa University Hospital, Pisa, Italy

15. Epilepsy Center, AOU Città della Salute e della Scienza di Torino, Turin, Italy

16. "Rita Levi Montalcini” Department of Neurosciences, University of Turin, Turin, Italy Italy

17. Epilepsy Center, UOC Neurology, AULSS 8 Vicenza, Vicenza,

18. Department Neurology 2, Careggi University Hospital, Florence, Italy

19. "C. Munari" Epilepsy Surgery Centre, Niguarda Hospital, Milan, Italy

20. Dipartimento di Neurologia, Neurofisiopatologia, Neurobiologia, Università Campus Bio-Medico, Roma, Italy

21. Neurology Unit, Maggiore Hospital, ASST Lodi, Lodi, Italy Italy

22. Circolo Hospital, Fondazione Macchi-ASST Settelaghi, Varese,

23. USL Central Tuscany, Neurology Unit, Nuovo Ospedale Santo Stefano, Prato, Italy

24. Neurology Unit, Maggiore Hospital, ASST Lodi, Lodi, Italy
25. San Gerardo Hospital, ASST Monza, Italy

26. Complex Structure of Neurology, SS Annunziata Hospital, Taranto, Italy

27. IRCCS Medea Scientific Institute, Epilepsy Unit, Conegliano, Treviso, Italy

28. Neurology Unit, Giovanni Paolo II Hospital, Ragusa, Italy

29. Department of Neurology, Hospital of Merano (SABESASDAA), Merano-Meran, Italy

30. Dipartimento di Neurologia, Ospedale Santa Maria della Misericordia, Università di Perugia, Perugia, Italy

31. Neurology Unit, AOU Ferrara, Ferrara, Italy

32. Neurology Unit, San Giovanni Bosco Hospital, Turin, Italy

33. Institute of Clinical Neurophysiology, Department of Neuroscience, Policlinico Riuniti, Foggia, Italy

34. Department of Life, Health, and Environmental Sciences, University of L'Aquila, Epilepsy Center, Ospedale San Salvatore, L'Aquila, Italy

35. Presidio Sanitario Major, Istituti Clinici Scientifici Maugeri, Turin, Italy

36. Neurodegenerative Disorders Unit, Azienda Sanitaria Provinciale di Caltanissetta, Caltanissetta, Italy

37. Department of Neuroscience, Imaging and Clinican Science, "G. D’Annunzio" University of Chieti-Pescara, Italy

38. Neurology Unit, "Perrino" Hospital, Brindisi, Italy

39. Oasi Research Institute- IRCCS, Troina, Italy

40. IRCCS Fondazione Don Carlo Gnocchi, Florence, Italy

41. Division of Clinical Neurophysiology and Epilepsy Center, IRCCS Ospedale Policlinico San Martino, Genova, Italy

42. Department of Neurosciences, University of Padua, Padua, Italy 43. University of Cagliari, Cagliari, Italy

44. Dipartimento di neurologia, Università "Magna Græcia", Catanzaro, Italy

45. Neurology Unit, Department of Cardio-neuro-vascular Sciences, San Donato Hospital, Arezzo, Italy

46. Department of Medical and Surgical Sciences and Advanced Technologies "G.F. Ingrassia", Section of Neurosciences, University of Catania, Catania, Italy

47. Department ofClinical Neurophysiology, Cardarelli Hospital, Naples, Italy

48. Epilepsy Centre, Neurology Unit, Department of Systems Medicine, Policlinico Tor Vergata, University of Rome "Tor Vergata", Rome, Italy

49. Department of Biomedical, Metabolic and Neural Science, University of Modena and Reggio Emilia, Modena, Italy

50. Clinical Neurology Department of Neurosciences University Hospital S. Maria della Misericordia, Udine, Italy

51. Neurology Unit Department of Neurosciences University Hospital S. Maria della Misericordia, Udine, Italy

52. Neurology Unit, ASST Rhodense, Milan, Italy

53. Dipartimento di neurologia, Ospedale San Filippo Neri, Roma

54. Neurology and epilepsy unit Humanitas Gradenigo Hospital, Turin, Italy

55. Neurology Unit, Department of Clinical and Experimental Medicine, University of Messina, Italy

56. Neurology Unit, Cutroni-Zodda Hospital, Barcellona, Messina, Italy

57. IRCCS Centro Neurolesi Bonino-Pulejo, Messina, Italy

58. AOU Sassari, Sassari, Italy

59. Neurology Unit, ASST Papa Giovanni XXIII, Bergamo, Italy

60. Epilepsy Outpatient Clinic for Adults. "A. Cardarelli” Hospital, Naples, Italy

61. Dipartimento di neurologia e neurofisiopatologia, Aurelia Hospital, Roma

62. Neurology Clinic, ASUGI, Trieste, Italy

63. Epilepsy Center, IRCCS Mondino Foundation, Pavia, Italy

64. Neurology and Stroke Unit, P.O. ARNAS-Civico, Palermo, Italy 
Open Access This article is licensed under a Creative Commons Attribution-NonCommercial 4.0 International License, which permits any non-commercial use, sharing, adaptation, distribution and reproduction in any medium or format, as long as you give appropriate credit to the original author(s) and the source, provide a link to the Creative Commons licence, and indicate if changes were made. The images or other third party material in this article are included in the article's Creative Commons licence, unless indicated otherwise in a credit line to the material. If material is not included in the article's Creative Commons licence and your intended use is not permitted by statutory regulation or exceeds the permitted use, you will need to obtain permission directly from the copyright holder. To view a copy of this licence, visit http://creativecommons.org/licenses/by-nc/4.0/. 